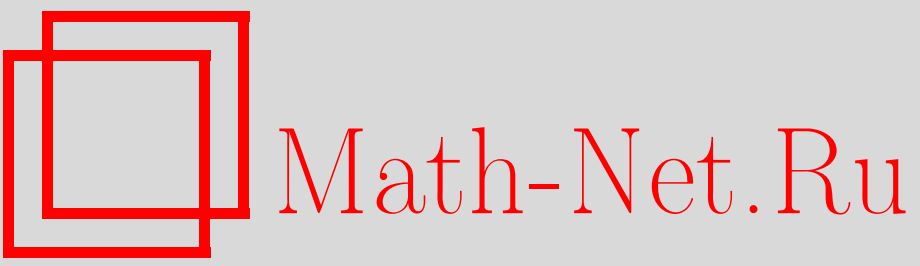

О. В. Солонуха, О нелинейной параболической задаче с препятствием, УМН, 2004, том 59, выпуск 3, 181-182

DOI: https://doi.org/10.4213/rm754

Использование Общероссийского математического портала Math-Net.Ru подразумевает, что вы прочитали и согласны с пользовательским соглашением

http://www.mathnet.ru/rus/agreement

Параметры загрузки:

IP: 3.81 .55 .215

26 апреля 2023 г., 18:03:52 


\title{
О НЕЛИНЕЙНОЙ ПАРАБОЛИЧЕСКОЙ ЗАДАЧЕ С ПРЕПЯТСТВИЕМ
}

\author{
О. В. Солонуха
}

Задачи с препятствием изучались в [1]-[3] и др. В данной работе критерий существования обобщенного решения сформулирован для более широкого класса существенно нелинейных задач.

Пусть $m, n \in \mathbb{N}, \Omega \subset \mathbb{R}^{n}$ - ограниченная связная область класса $C^{m, 1}$ с границей $\partial \Omega, p \in$ $[2, \infty), 1 / q+1 / p=1 ; Q_{T}=\Omega \times(0, T), \Gamma_{T}=\partial \Omega \times(0, T)$. Обозначим через $\partial_{t}: \operatorname{Dom}\left(\partial_{t}\right) \subset X=$ $L_{p}(0, T ; \stackrel{\circ}{W} \underset{p}{m}(\Omega)) \rightarrow L_{q}\left(0, T ; W_{q}^{-m}(\Omega)\right)=X^{*}$ оператор дифференцирования по $t$. Рассмотрим задачу с препятствием

$$
\left\{\begin{array}{l}
f \leqslant \partial_{t} y+A y, \quad y \geqslant \psi,(y-\psi)\left(\partial_{t} y+A y-f\right)=0 \text { п.в. на } Q_{T}, \\
y(0, x)=y_{0}(x) \text { п.в. на } \Omega, \frac{\partial^{k} y}{\partial \nu^{k}}=0 \text { п.в. на } \Gamma_{T} \forall k \leqslant m-1,
\end{array}\right.
$$

где $y_{0} \in L_{2}(\Omega)$; препятствие $\psi \in L_{p}\left(0, T ; W_{p}^{m}(\Omega)\right)$ таково, что $\psi \leqslant 0$ почти всюду на $\Gamma_{T}$, $\psi(0, x) \leqslant y_{0}(x)$ почти всюду на $\Omega, \partial_{t} \psi \in L_{q}\left(0, T ; W_{q}^{-m}(\Omega)\right) ; f=\sum_{|\alpha| \leqslant m}(-1)^{|\alpha|} D^{\alpha} f_{\alpha}$, $f_{\alpha} \in L_{q}\left(Q_{T}\right) ; \alpha=\left(\alpha_{1}, \ldots, \alpha_{n}\right)$ - мультииндекс, $|\alpha|=\alpha_{1}+\cdots+\alpha_{n} ; D^{\alpha} y=\frac{\partial^{\alpha_{1}}}{\partial x_{1}^{\alpha_{1}}} \ldots \frac{\partial^{\alpha_{n}}}{\partial x_{n}^{\alpha_{n}}} y$; для каждого $\xi \in X=L_{p}(0, T ; \stackrel{\circ}{W} \underset{p}{m}(\Omega))$ нелинейный оператор $A: X \rightarrow X^{*}$ задан выражением

$$
\langle A y, \xi\rangle=\sum_{|\alpha| \leqslant m} \int_{Q_{T}} A_{\alpha}\left(t, x, y, D^{1} y, \ldots, D^{m} y\right) D^{\alpha} \xi d x d t
$$

$D^{k} y$-набор всех производных порядка $k$ для натурального $k=\overline{1, m}$. Обозначим через $\mathrm{M}_{\text {и }} \mathrm{M}^{\prime}$ число различных мультииндексов $\alpha$ таких, что $0<|\alpha| \leqslant m$ и $0<|\alpha| \leqslant m-1$ соответственно.

Сформулируем условия на функции $A_{\alpha}$.

А1. Условие интегрируемости. Функции $A_{\alpha}$ измеримы по $(t, x) \in Q_{T}$ и непрерывны по остальным аргументам. Кроме того, для почти всех $(t, x) \in Q_{T}$ и всех $\xi=\left(\xi_{0}, \ldots, \xi_{\mathrm{M}}\right) \in \mathbb{R}^{\mathrm{M}+1}$

$$
\left|A_{\alpha}(t, x, \xi)\right| \leqslant c_{1} \sum_{0 \leqslant j \leqslant M}\left|\xi_{j}\right|^{p-1}+h_{\alpha}(t, x), \quad|\alpha| \leqslant m,
$$

где $c_{1}>0, h_{\alpha} \in L_{q}\left(Q_{T}\right)$.

А2. Условие эллиптичности. Для почти всех $(t, x) \in Q_{T}$ и любых $\xi, \xi^{\prime} \in \mathbb{R}^{\mathrm{M}+1}$ таких, что $\xi=(\omega, \zeta), \xi^{\prime}=(\omega, \eta), \omega \in \mathbb{R}^{\mathrm{M}^{\prime}+1}$, выполняется неравенство

$$
\sum_{|\alpha|=m}\left(A_{\alpha}(t, x, \omega, \zeta)-A_{\alpha}(t, x, \omega, \eta)\right)\left(\zeta_{\alpha}-\eta_{\alpha}\right)>0, \text { если } \eta \neq \zeta .
$$

А3. Условие коэрцитивности. Для почти всех $(t, x) \in Q_{T}$ и любых $\xi \in \mathbb{R}^{\mathrm{M}+1}$ справедлива оценка

$$
\sum_{|\alpha| \leqslant m} A_{\alpha}(t, x, \xi) \xi_{\alpha} \geqslant c_{2} \sum_{|\alpha|=m}\left|\xi_{\alpha}\right|^{p}-g(t, x)
$$

где $c_{2}>0$ и $g \in L_{1}(Q)$.

Сильныц обобщенным решением задачи (1) назовем элемент

$$
y \in K=\left\{z \in L_{p}(0, T ; \stackrel{\circ}{W} \underset{p}{m}(\Omega)):\left.z\right|_{t=0}=y_{0} \text { п.в. на } \Omega, z \geqslant \psi \text { п.в. на } Q_{T}\right\},
$$

(C) О.В. Солонуха 2004 
удовлетворяющий вариационному неравенству

$$
\left\langle\partial_{t} y+A y, \xi-y\right\rangle \geqslant \sum_{|\alpha| \leqslant m} \int_{Q_{T}} f_{\alpha} D^{\alpha}(\xi-y) d x d t \quad \forall \xi \in K .
$$

Заметим, что при этом $\partial_{t} y \in L_{q}\left(0, T ; W_{q}^{-m}(\Omega)\right)$.

Назовем последовательность $\left\{\left(y_{k}, A y_{k}\right)\right\} \omega_{W}$-последовательностью, если $y_{n} \rightarrow y$ слабо в $X, \partial_{t} y_{k} \rightarrow \partial_{t} y$ слабо в $X^{*}$ и $\overline{\lim }_{k \rightarrow \infty}\left\langle A y_{k}, y_{k}-y\right\rangle \leqslant 0$. Оператор $A: \operatorname{Dom}(A) \subset X \rightarrow$ $X^{*}$ обладает свойством $\left(S_{+}\right)$относительно $\partial_{t}$, если для любой $\omega_{W}$-последовательности $y \in \operatorname{Dom}(A), y_{k} \rightarrow y$ в $X$ и $A y_{k} \rightarrow A y$ слабо в $X^{*}$. Оператор $A: \operatorname{Dom}(A) \subset X \rightarrow X^{*}$ псевдомонотонен на $\operatorname{Dom}\left(\partial_{t}\right)$, если для любой $\omega_{W}$-последователшности справедлива оценка $\underline{\lim }_{k \rightarrow \infty}\left\langle A y_{k}, \xi-y_{k}\right\rangle \geqslant\langle A(y), \xi-y\rangle$ для всех $\xi \in X$. Оператор $A: \operatorname{Dom}(A) \subset X \rightarrow X^{*}$ коэриитивен, если $\lim _{\|y\|_{X} \rightarrow \infty}\|y\|_{X}^{-1}\langle A y, y\rangle=+\infty$.

Теорема 1. Пусть коэффициенты $A_{\alpha}$ удовлетворяют условиям A1-A3. Тогда оператор $A: L_{p}\left(0, T ; \stackrel{\circ}{W}_{p}^{m}(\Omega)\right) \rightarrow L_{q}\left(0, T ; W_{q}^{-m}(\Omega)\right)$, заданный формулой (2), ограничен, псевдомонотонен на $\operatorname{Dom}\left(\partial_{t}\right)$, имеет свойство $\left(S_{+}\right)$относительно $\partial_{t}$ и коэрцитивен.

Теорема 2. Пусть коэффициенты $A_{\alpha}$ удовлетворяют условиям А1-А ча с препятствием (1) имеет непустое, компактное множество сильных обобщенных решений.

Доказательства теорем 1, 2 основаны на методах [4].

\section{СПИСОК ЛИТЕРАТУРЫ}

[1] Д. Е. Апушкинская, Н. Н. Уральцева, Х. Шахголян // Алгебра и анализ. 2002. Т. 14. №1. С. 3-25. [2] А. А. А рхипова // Проблемы матем. анализа. Вып. 9. Л.: Изд-во ЛГУ, 1984. C. 149-157. [3] G. M. Troianiello // Manuscripta Math. 1979. V. 29. № 2-4. P. 353-384. [4] O. V. Solonukha // Mitt. Math. Sem. Giessen. 2003. № 251.

Киевский политехнический институт

E-mail: olesia@solonukha.mtu-net.ru, skubachevskii@yandex.ru
Принято редколлегией 01.04 .2004 